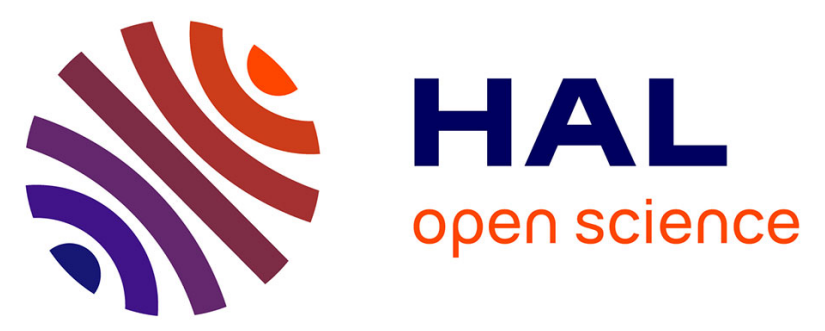

\title{
Situation sanitaire et parcours de soins des personnes âgées en milieu rural africain. Une étude à partir des données du suivi de population de Mlomp (Sénégal)
}

Géraldine Duthé, Gilles Pison, Raphäl Laurent

\section{- To cite this version:}

Géraldine Duthé, Gilles Pison, Raphaël Laurent. Situation sanitaire et parcours de soins des personnes âgées en milieu rural africain. Une étude à partir des données du suivi de population de Mlomp (Sénégal). Autrepart - Revue de sciences sociales au Sud, 2010, Vieillir au Sud, 53, pp.167-187. 10.3917/autr.053.0167 . hal-03130795

\section{HAL Id: hal-03130795 \\ https://hal.science/hal-03130795}

Submitted on 3 Feb 2021

HAL is a multi-disciplinary open access archive for the deposit and dissemination of scientific research documents, whether they are published or not. The documents may come from teaching and research institutions in France or abroad, or from public or private research centers.
L'archive ouverte pluridisciplinaire HAL, est destinée au dépôt et à la diffusion de documents scientifiques de niveau recherche, publiés ou non, émanant des établissements d'enseignement et de recherche français ou étrangers, des laboratoires publics ou privés. 


\title{
Situation sanitaire et parcours de soins des personnes âgées en milieu rural africain Une étude à partir des données du suivi de population de Mlomp (Sénégal)
}

\author{
Géraldine Duthé*, Gilles Pison**, Raphaël Laurent***
}

\section{La situation sanitaire des personnes âgées en Afrique}

La situation sanitaire des personnes âgées en Afrique est peu connue et les recherches dans ce domaine, récentes (Velkoff et Kowal, 2007 ; National Research Council, 2006 ; Kowal et al., 2000 ; 2002). L'étude de la mortalité adulte se restreint généralement aux moins de 60 ans (Hill, 1999 ; Timæus and Jasseh, 2004) et celle des plus âgés pose d'importants problèmes de mesure, notamment de fiabilité des âges déclarés. En milieu rural où on retrouve la plupart des personnes âgées (Tabutin, 1987; Schoumaker, 2000), l'accès aux soins est d'autant plus difficile qu'il s'agit de personnes peu mobiles. En outre, les personnes âgées préféreraient recourir à la médecine traditionnelle (Massengo, 2002) et, malades, seraient plus enclines à rester chez elles pour mourir près de leurs proches (Thomas, 1994). L'offre de soins dans les infrastructures sanitaires, toujours centrée sur la santé reproductive, n'est pas adaptée aux problèmes de santé des adultes et des personnes âgées en particulier (Shrestha, 2000 ; Attias-Donfut et Rosenmayr, 1994 ; Kouamé, 1990).

En 2005-2010, l'espérance de vie à la naissance est estimée à 51,5 ans en Afrique subsaharienne et les personnes âgées de 60 ans auraient encore en moyenne une quinzaine d'années à vivre (14,8 ans pour les hommes et 16,6 ans pour les femmes), soit quatre années de moins que la moyenne mondiale (Nations Unies, 2009). Il existe probablement une grande diversité de situations, notamment entre le monde urbain et le monde rural, ce dernier étant généralement caractérisé

\footnotetext{
* Chargée de recherche, Unité Mortalité, Santé, Épidémiologie, Institut national d'études démographiques, Centre Population et développement (CEPED - UMR INED/IRD/Université Paris Descartes).

** Directeur de recherche, Unité Mortalité, Santé, Épidémiologie, Institut national d'études démographiques, Centre Population et développement (CEPED - UMR INED/IRD/Université Paris Descartes).

*** Ingénieur d'études, Service des enquêtes, Institut national d'études démographiques.
} 
par de moins bonnes conditions socio-économiques et sanitaires impliquant des niveaux de mortalité plus élevés. La connaissance des causes de décès reste également très fragmentaire. Comme dans les pays du Nord, les problèmes de santé des personnes âgées sont aussi liés aux maladies cardiovasculaires, aux cancers et autres maladies de dégénérescence, pour la plupart chroniques et non transmissibles (Prost, 2000). Mais ces «pathologies de la sénescence sont d'autant plus graves qu'elles surviennent sur un fond de fragilité dû aux carences nutritionnelles et à de multiples affections » (Attias Donfut et Rosenmayr, 1994, p. 14). En Afrique subsaharienne, les personnes âgées sont toujours victimes de maladies infectieuses (tuberculose, sida et infections digestives chroniques) ainsi que de troubles nutritionnels (Kahn et al., 2006 ; Sankoh et al., 2003 ; Massengo, 2002 ; Ka-Cisse et Sow, 1995 ; Centre international de gérontologie sociale, 1985).

Les sites de suivi démographique recueillent les événements démographiques qui surviennent au sein d'une population délimitée localement, par le biais d'enquêtes à passages répétés faisant suite à un recensement initial (Pison, 2005). La méthode d'autopsie verbale permet d'identifier les causes de décès : elle consiste à interroger les proches de l'individu décédé pour recueillir des informations sur la maladie, le traitement et les symptômes. Le médecin est ensuite requis pour l'interprétation des informations recueillies et la détermination des causes probables de décès (Fauveau, 2006). Un intérêt particulier est porté à la validité de la méthode d'autopsie verbale : celle-ci dépend du processus morbide ayant conduit au décès et du protocole d'enquête mis en place (Garenne et Fauveau, 2006). Les sites de suivi démographique contribuent à une meilleure connaissance démographique de la situation sanitaire, variable selon les pays et les régions (Adjuik et al., 2006). Ils permettent en effet de décrire les populations, de mesurer précisément les niveaux de mortalité et fournissent des données sur les causes de décès non disponibles par ailleurs, surtout aux âges adultes (Kaufman et al., 1997). Toutefois, peu de résultats concernant les plus âgés sont publiés.

\section{Une étude à partir des données du suivi de population de Mlomp, en milieu rural sénégalais}

La question de l'état de santé des personnes âgées en Afrique et de leur prise en charge devient préoccupante (Antoine, 2007; National Research Council, 2006 ; Zimmer et Dayton, 2005). La faible connaissance de la situation sanitaire des plus âgés accentue les difficultés à mettre en place un système de soins efficace et accessible à la population dans son ensemble (Schieber et Maeda, 1999). Dans cette étude, nous cherchons à appréhender la situation sanitaire des personnes âgées en milieu rural africain en nous appuyant sur les données du suivi démographique et sanitaire de Mlomp au Sénégal. Bien qu'elles aient leurs limites pour étudier les plus âgés de la population, les données de suivi fournissent des informations sanitaires sur les personnes âgées non disponibles par ailleurs. 
Au Sénégal, la prévalence du VIH/sida se maintient à un niveau relativement bas, autour de $1 \%$ parmi les 15-49 ans (ONUSIDA, 2008), mais les progrès sanitaires sont lents et les niveaux de mortalité élevés. L'espérance de vie y est estimée à 54 et 57 ans pour les hommes et les femmes pour 2005-2010; la population est très jeune, et seulement $4 \%$ auraient plus de 60 ans (Nations Unies, 2009). Des différences importantes s'observent entre milieu rural et milieu urbain. Ainsi, la mortalité avant 5 ans est près de deux fois plus élevée dans les campagnes avec en moyenne 16 décès sur 100 naissances ; du point de vue économique, la moitié des ménages sénégalais vivent sous le seuil de pauvreté mais c'est le cas de $65 \%$ des ménages en milieu rural (Ndiaye et Ayad, 2006).

S'appuyant sur les recommandations de l'OMS, le Sénégal, qui fait partie des pays les moins avancés économiquement d'après les critères des Nations Unies, a depuis son indépendance mis l'accent sur les soins de santé primaire et de proximité considérés comme prioritaires depuis la conférence de Alma Ata en 1978. Ce dispositif est principalement centré sur la santé de la reproduction et des enfants même s'il s'est récemment diversifié, selon les directives des Objectifs du millénaire pour le développement. Pour améliorer l'accès aux soins primaires en zone rurale, le système de soins est décentralisé avec la formation d'agents de santé et de matrones, l'ouverture de cases de santé, de postes de santé et de maternités, et l'implication des communautés villageoises dans les actions sanitaires. Un centre de santé dirigé par un médecin, a été ouvert dans chaque chef-lieu de département. Ces centres de santé constituent les hôpitaux de référence pour les postes de santé du département gérés par des infirmiers.

Au Sénégal, la population de Mlomp est suivie annuellement depuis son recensement en 1985. Les causes de décès y sont déterminées avec la méthode d'autopsie verbale, complétée par les informations médicales provenant des infras-

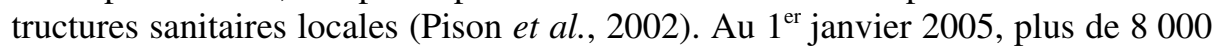
personnes étaient suivies à Mlomp. Les habitants vivent dans des ménages regroupés en concessions familiales, selon un système patrilinéaire. D'ethnie diola, les habitants sont majoritairement de religion animiste ou catholique. La culture $\mathrm{du}$ riz est la principale ressource locale et la plupart des jeunes femmes et des hommes migrent pour gagner de l'argent, surtout durant la saison sèche, période de faible activité agricole. Avec un dispensaire et une maternité qui existent depuis les années 1960, le dispositif sanitaire est bien développé pour une zone rurale et a permis une forte diminution de la mortalité avant 5 ans (Pison et al., 1993). La couverture vaccinale des enfants est excellente et toutes les femmes accouchent en maternité (Duthé, 2008). Sur la période 1985-2004, l'espérance de vie à la naissance atteint 61 ans, ce qui est plutôt élevé pour une zone rurale. L'écart important entre les sexes - 65 ans pour les femmes et 57 ans pour les hommes résulte en partie d'une mortalité maternelle relativement basse et d'une mortalité violente relativement élevée chez les hommes (Pison et al., 2000 ; 2005).

À partir des indicateurs démographiques énoncés, on peut donc s'attendre à une proportion de personnes âgées relativement élevée, pour lesquelles on dispose 
d'informations sur leur mortalité (niveau et causes de décès) depuis 1985. Mlomp est le seul des trois sites de suivi démographique ruraux du Sénégal à disposer de causes probables de décès au-delà de 60 ans depuis le début du suivi. En outre, les histoires des maladies racontées par les proches fournissent des informations sur le recours aux soins avant la survenue du décès. Plusieurs hypothèses soustendent cette recherche, la première est que dans le contexte où les familles constituent la première aide aux soins des personnes âgées, le rôle de l'entourage est crucial dans les décisions de soins mais aussi dans l'attention qui leur est portée. On suppose que la prise en charge sanitaire est différente selon le sexe, selon le statut des hommes et des femmes, et l'âge, avec l'entrée dans la vieillesse. Nous pensons que malgré un recours aux services de santé notablement fréquent dans la population, notamment pour les enfants et les femmes enceintes, de nombreux facteurs freinent la consultation des personnes âgées auprès de ces services de santé.

\section{Population des plus de 60 ans}

Parmi la population suivie au $1^{\text {er }}$ janvier 2005 à Mlomp, plus de $10 \%$ des habitants sont âgés de plus de 60 ans. Sans tenir compte des habitants absents à cette date assimilés ici aux migrants ${ }^{1}$, cette proportion atteint $15 \%$. Les personnes âgées de plus de 60 ans constituent donc une part relativement importante de la population de Mlomp. On compte un peu plus de femmes que d'hommes après 60 ans mais elles sont en proportion plus nombreuses $(59 \%)$ après 80 ans ; la part des plus de 80 ans restant globalement très faible (1\%) (Tableau 1).

Tableau 1 - Population âgée de plus de 60 ans (Mlomp, janvier 2005)

\begin{tabular}{l|c|c|c|c|c|c|c|c|c}
\hline & \multicolumn{3}{|c|}{ Effectifs } & \multicolumn{3}{c|}{$\begin{array}{c}\text { Répartition } \\
\text { par groupe d'âge }\end{array}$} & \multicolumn{3}{c}{ Répartition par sexe } \\
& Hom. & Fem. & Ens. & Hom. & Fem. & Ens. & Hom. & Fem. & Ens. \\
\hline $\begin{array}{l}60 \text { ans et } \\
\text { plus }\end{array}$ & 401 & 437 & 838 & 9,7 & 11,3 & 10,5 & 47,9 & 52,1 & 100,0 \\
\hline $\begin{array}{l}\text { dont } 80 \\
\text { ans et plus }\end{array}$ & 31 & 44 & 75 & 0,8 & 1,1 & 0,9 & 41,3 & 58,7 & 100,0 \\
\hline \begin{tabular}{l} 
Tous âges \\
\hline
\end{tabular} & 4124 & 3883 & 8007 & 100,0 & 100,0 & 100,0 & 51,5 & 48,5 & 100,0 \\
\hline
\end{tabular}

Source : base de données de Mlomp.

1. Du fait du calendrier de la collecte qui intervient en janvier en période de saison sèche, la quasitotalité des personnes absentes au moment de la collecte sont partis en migration. 
Du point de vue de leur statut dans le ménage, la quasi-totalité des hommes de plus de 60 ans sont chefs de leur ménage ; les femmes se répartissent entre ce statut $(47 \%)$ et celui d'épouse du chef (44\%). Elles sont aussi plus nombreuses à vivre dans le ménage comme autre adulte, généralement celui d'un de leurs enfants (Tableau 2).

Tableau 2 - Personnes âgées de plus de 60 ans selon leur statut dans le ménage (Mlomp, janvier 2005)

\begin{tabular}{l|c|c}
\hline \multicolumn{1}{c|}{ Statut dans le ménage } & Hommes & Femmes \\
\hline Chef de ménage & 95,0 & 47,1 \\
\hline Épouse du chef de ménage & - & 43,9 \\
\hline Autre adulte & 5,0 & 8,9 \\
\hline Ensemble & 100,0 & 100,0 \\
\hline Effectifs & 401 & 437 \\
\hline
\end{tabular}

Source : base de données de Mlomp.

Les femmes chefs de ménage pourraient être plus isolées que les épouses de chef de ménage, ces dernières étant forcément mariées contrairement aux premières. Globalement après 60 ans, les hommes sont d'ailleurs bien plus fréquemment mariés $(76 \%)$ que les femmes $(46 \%)$, cette distinction est très nette après 80 ans où la plupart des femmes sont veuves alors que la moitié des hommes sont encore mariés (Tableau 3).

Tableau 3 - Personnes âgées de plus de 60 ans selon leur situation matrimoniale (Mlomp, janvier 2005)

\begin{tabular}{l|c|c|c|c|c|c}
\hline & \multicolumn{3}{|c|}{ Hommes } & \multicolumn{3}{c}{ Femmes } \\
\hline & 60-79 ans & $\begin{array}{c}\mathbf{8 0} \text { ans } \\
\text { et }+\end{array}$ & $\begin{array}{c}60 \text { ans } \\
\text { et }+\end{array}$ & $\mathbf{6 0 - 7 9}$ ans & $\begin{array}{c}\mathbf{8 0} \text { ans } \\
\text { et + }\end{array}$ & $\begin{array}{c}\text { 60 ans } \\
\text { et + }\end{array}$ \\
\hline Marié-e & 78,1 & 51,6 & 76,1 & 50,5 & 6,7 & 46,0 \\
\hline Divorcé-e & 8,6 & 16,1 & 9,2 & 3,3 & 4,4 & 3,4 \\
\hline Veuf-ve & 10,5 & 32,3 & 12,2 & 44,2 & 88,9 & 48,7 \\
\hline Célibataire/inconnu & 2,7 & 0,0 & 2,5 & 2,0 & 0,0 & 1,8 \\
\hline Total & 100,0 & 100,0 & 100,0 & 100,0 & 100,0 & 100,0 \\
\hline Effectifs & 370 & 31 & 401 & 394 & 45 & 439 \\
\hline
\end{tabular}

Source : base de données de Mlomp. 
Dans les pays africains, très peu de personnes âgées vivent seules (Locoh et Makdessi, 2002), l'isolement est donc un phénomène plutôt rare. Néanmoins, l'exode rural entraîne des conséquences sur les conditions de vie des personnes les plus âgées, notamment en termes de distanciation. Les femmes, plus nombreuses, plus souvent veuves et ne bénéficiant pas du prestige de l'homme vieillard seraient plus fragilisées par cette situation (Renaut, 1994). Pour étayer ces hypothèses, nous nous sommes intéressés à la taille des ménages des personnes âgées à Mlomp en distinguant les personnes présentes au village de l'ensemble des personnes suivies (Tableau 4). En janvier 2005, les habitants vivent dans des ménages qui comptent en moyenne 9 membres. En ne tenant compte que des personnes présentes à cette date, cette moyenne se maintient à plus de 6 . Avec un nombre moyen de personnes présentes autour de 4,5, la taille des ménages dans lesquels vivent les seniors est donc globalement moins élevée. Tous âges confondus, seulement $1 \%$ des hommes et des femmes vivent seuls dans leur ménage, cette proportion augmente mais reste globalement assez faible si on ne tient compte que des individus présents. Avec les migrations, les personnes âgées vivant en milieu rural africain auraient aussi souvent la charge de leurs petitsenfants (Zimmer et Dayton, 2005). Si l'on ne tient compte que des adultes de plus de 15 ans, la proportion de personnes âgées vivant seules dans leur ménage augmente mais très légèrement. À Mlomp, peu de seniors vivent donc seuls avec des enfants dans le ménage.

Environ $13 \%$ des hommes et des femmes de plus de 60 ans (plus d'un quart parmi les plus de 80 ans) vivent seuls, mais à l'exception du veuvage bien plus important chez les femmes, ces résultats ne révèlent pas de différence entre les hommes et les femmes. Pour autant, il est difficile de vérifier quantitativement l'isolement des personnes âgées, qui nécessite une étude plus fine des liens entretenus avec les proches dans le ménage et en dehors, notamment dans la concession.

\section{Mortalité des plus de 60 ans}

Le suivi apporte une précision de la datation des naissances et des décès observés. Cependant, la date de naissance des personnes présentes au recensement reste une estimation d'autant moins fiable que l'individu est âgé. À Mlomp, si les sources disponibles localement ont été mobilisées au moment du recensement, l'âge des plus anciens reste incertain. De plus, les effectifs des survivants sont faibles au-delà d'un certain âge, et l'observation de la mortalité devient délicate. En modélisant la mortalité après 80 ans, nous avons estimé l'espérance de vie à 60 ans de 16,5 ans pour l'ensemble de la population, avec un écart de plus de 3 ans entre les hommes (14,9) et les femmes (18,3) (Duthé et al., 2009).

À Mlomp, les autopsies verbales se font au moment de la collecte annuelle, dans un délai allant de la fin de la période de deuil à une année. Elles sont menées par des enquêteurs originaires du village, permettant une meilleure compréhension 
Tableau 4 - Taille des ménages selon le sexe et le groupe d'âge (Mlomp, janvier 2005)

\begin{tabular}{l|c|c|c|c}
\hline & \multicolumn{2}{|c|}{ Hommes } & \multicolumn{2}{c}{ Femmes } \\
\hline & $\begin{array}{c}\text { Ensemble des } \\
\text { membres }\end{array}$ & $\begin{array}{c}\text { Présents au } \\
1^{\text {er }} \text { janvier }\end{array}$ & $\begin{array}{c}\text { Ensemble des } \\
\text { membres }\end{array}$ & $\begin{array}{c}\text { Présents au } \\
1^{\text {er }} \text { janvier }\end{array}$ \\
\hline
\end{tabular}

Nombre moyen de personnes par ménage

\begin{tabular}{l|l|l|l|l}
\hline $60-79$ ans & 7,6 & 4,7 & 6,9 & 4,4 \\
\hline 80 ans et plus & 4,6 & 3,3 & 5,8 & 3,7 \\
\hline 60 ans et plus & 7,3 & 4,6 & 6,8 & 4,3 \\
\hline Tous âges & 8,9 & 6,4 & 8,7 & 6,9 \\
\hline
\end{tabular}

Proportion de personnes seules dans un ménage (en \%)

\begin{tabular}{l|c|c|c|c}
\hline $60-79$ ans & 4,9 & 10,9 & 5,1 & 9,1 \\
\hline 80 ans et plus & 9,7 & 25,8 & 6,7 & 25,6 \\
\hline 60 ans et plus & 5,2 & 12,2 & 5,2 & 10,8 \\
\hline Tous âges & 1,5 & 3,5 & 0,8 & 2,4 \\
\hline
\end{tabular}

Proportion de personnes vivant dans un ménage sans un autre adulte âgé de plus de 15 ans $($ en $\%)$

\begin{tabular}{l|l|l|l|l}
\hline $60-79$ ans & 5,1 & 12,1 & 6,6 & 10,7 \\
\hline 80 ans et plus & 9,7 & 25,8 & 6,7 & 27,9 \\
\hline 60 ans et plus & 5,5 & 13,3 & 6,6 & 12,5 \\
\hline Tous âges & 2,1 & 6,6 & 2,1 & 7,4 \\
\hline
\end{tabular}

Source : base de données de Mlomp.

linguistique et socioculturelle, et une relation de confiance indispensable au bon déroulement de l'entretien ${ }^{2}$ (Chandramohan et al., 2005). Ce sont les enquêteurs qui veillent à trouver la personne la plus apte à répondre aux questions sur l'histoire de la maladie et des symptômes. Depuis 1985, le même questionnaire est utilisé pour tous les décès. Après l'identification de la personne décédée et du répondant, ce dernier déclare la cause qu'il suppose être à l'origine du décès, les éventuels traitements que le malade a reçus et raconte la maladie. Le questionnaire passe ensuite en revue toute une série de symptômes à détailler s'ils sont signalés par le répondant (Duthé, 2008). L’apport des informations médicales recueillies

2. Avant de commencer le questionnaire, l'enquêteur explique les objectifs de l'enquête et demande l'accord oral du répondant. 
auprès des institutions sanitaires locales contribue grandement à augmenter la validité de la méthode. Le protocole de détermination des causes de décès a sensiblement été le même jusqu'en 2003 : un seul médecin déterminait les causes de décès en se rendant à Mlomp à la fin du passage annuel pour consulter les différents registres tenus par l'infirmière du dispensaire ${ }^{3}$ et compléter les autopsies verbales. Depuis 2004, les informations médicales disponibles sont saisies, l'ensemble est emmené à Dakar où deux médecins diagnostiquent. Les causes de décès sont codées selon la $9^{e}$ révision de la Classification internationale des maladies (OMS, 1977). Seule la cause principale du décès est saisie. Malgré l'apport des informations médicales, sur la période 1985-2004, $35 \%$ des décès survenus entre 60 et 80 ans sont de cause mal définie et cette proportion atteint $47 \%$ après 80 ans $^{4}$ (Tableau 5). En outre, les décès de cause inconnue ou ceux liés à la sénilité constituent la moitié des décès mal définis avant 80 ans et les deux tiers au-delà de cet âge. Cette forte proportion de décès de cause mal définie rend caduc un effort d'estimation de taux de mortalité par cause après 60 ans. Nous nous en tenons donc ici à la distribution selon les diagnostics. Sans tenir compte des mal définis, les principales causes de décès sont les maladies cardiovasculaires, les maladies infectieuses, les tumeurs avant 80 ans, et les maladies de l'appareil respiratoire après 80 ans. Malgré l'importance des maladies non transmissibles, les maladies infectieuses et parasitaires - et notamment les tuberculoses, les infections intestinales comme le choléra et les septicémies -, conservent un poids conséquent.

Les causes de décès après 60 ans et en particulier après 80 ans restent donc mal connues, la maladie ayant conduit au décès est certainement plus complexe à discerner avec l'intervention de causes multiples et le rôle important de maladies non transmissibles plus difficiles à discerner par le biais des autopsies verbales. La distinction selon le sexe permet de voir que les décès féminins sont en proportion moins bien définis que ceux des hommes. Un des éléments explicatifs pourrait relever des comportements de recours aux soins.

\section{Parcours de soins des personnes décédées après 60 ans}

Dans la première partie du questionnaire d'autopsie verbale réalisé à la suite d'un décès, l'enquêteur invite le répondant, par une question ouverte, à raconter la maladie ayant conduit au décès en précisant les soins que le malade a reçus. Ces récits constituent une source d'informations sur les types de soins reçus par les malades avant leur décès. Elles restent toutefois limitées par le fait qu'elles ne sont par définition recueillies que pour les personnes décédées. Notre étude porte donc exclusivement sur les maladies fatales. Nous avons exploité les histoires de maladies provenant des autopsies verbales réalisées lors des trois enquêtes successives de 2002 à 2004, soit 240 récits de décès survenus sur la période de

3. Registre des consultations au dispensaire, registre des décès constatés par l'infirmière avec cause supposée, registre des consultations prénatales et registres des accouchements à la maternité.

4. Avant 60 ans, moins d'un quart des décès sont mal définis. 
février 2001 à février 2004 (les accidents et autres morts violentes ont été exclus) et parmi lesquels 134 sont survenus après 60 ans.

Tableau 5 - Causes de décès selon le groupe d'âge et le sexe (Mlomp, 1985-2004)

\begin{tabular}{l|c|c|c|c|c|c}
\hline & \multicolumn{3}{|c|}{ 60-79 ans } & \multicolumn{3}{c}{ 80 ans et + } \\
\hline \multicolumn{1}{c|}{$(\mathbf{e n ~ \% )}$} & Hom. & Fem. & Ens. & Hom. & Fem. & Ens. \\
\hline Tuberculose & 6 & 3 & 4 & 2 & 3 & 3 \\
\hline $\begin{array}{l}\text { Autres maladies } \\
\text { infect. et parasitaires }\end{array}$ & 7 & 7 & 7 & 10 & 9 & 9 \\
\hline $\begin{array}{l}\text { Tumeurs } \\
\text { Maladies de l'appareil }\end{array}$ & 22 & 24 & 23 & 25 & 23 & 24 \\
circulatoire & 15 & 13 & 14 & 3 & 3 & 4 \\
\hline $\begin{array}{l}\text { Maladies de l'appareil } \\
\text { respiratoire }\end{array}$ & 3 & 5 & 4 & 10 & 6 & 7 \\
\hline $\begin{array}{l}\text { Autres maladies et } \\
\text { morts violentes }\end{array}$ & 13 & 6 & 10 & 5 & 3 & 4 \\
\hline $\begin{array}{l}\text { Décès de cause mal } \\
\text { définie ou inconnue }\end{array}$ & 31 & 41 & 35 & 41 & 49 & 47 \\
\hline $\begin{array}{l}\text { dont symptômes } \\
\text { généraux }\end{array}$ & 5 & 8 & 6 & 13 & 13 & 13 \\
\hline $\begin{array}{l}\text { autres symptômes } \\
\text { sénilité }\end{array}$ & 11 & 10 & 11 & 0 & 5 & 4 \\
\hline $\begin{array}{l}\text { cause inconnue } \\
\text { personne-année) }\end{array}$ & 10 & 17 & 13 & 13 & 14 & 14 \\
\hline $\begin{array}{l}\text { Total } \\
\text { Nombre de décès }\end{array}$ & 336 & 231 & 567 & 61 & 134 & 195 \\
\hline Effectifs (en & 6850 & 7302 & 14152 & 352 & 831 & 1183 \\
\hline
\end{tabular}

Source : base de données de Mlomp.

Avant d'exposer les résultats de cette analyse, il est utile de présenter les différents modes de recours aux soins possibles à Mlomp et les motivations des individus à adopter un comportement plutôt qu'un autre. Si les acteurs du système de santé local entretiennent des liens étroits avec les habitants de Mlomp, notamment pour la santé maternelle et de l'enfant, les malades ne s'adressent pas 
systématiquement vers les services de santé. En cas d'épisode morbide, la fabrication de remèdes traditionnels, l'automédication, la consultation auprès d'un tradipraticien ou l'absence de recours sont aussi des comportements fréquents.

Généralement, la médecine traditionnelle apporte en plus d'une thérapeutique des éléments explicatifs d'ordre symbolique, religieux et social. Il n'y a pas de contradiction entre le fait de reconnaître une origine biomédicale à une maladie et celui d'en chercher la racine dans un domaine tout autre. Différentes stratégies pour se soigner sont donc possibles et celles-ci dépendent de beaucoup de facteurs, en particulier des symptômes, de l'âge du malade, de son statut social, des relations entretenues avec les soignants, de l'argent disponible et de la durée de la maladie (Duthé, 2006). Une maladie peut avoir une origine maléfique, dans un contexte particulier. Par exemple, selon la façon dont elle se manifeste et si l'entourage l'interprète comme tel. Les fétiches, les sacrifices ou les remèdes d'un guérisseur peuvent alors remédier à des problèmes de santé qui seraient liés à un totem fragile ou à un mauvais sort. À l'inverse, certains corpus de symptômes constituent des entités nosologiques populaires internes (Olivier de Sardan, 1999) qui regroupent des maladies identifiées par un nom spécifique, sans lien avec le surnaturel et très communes. Étant populaires, des traitements populaires y sont associés, souvent à base de plantes, constitués par la famille ou l'entourage. À Mlomp, lorsque le malade présente ce que les habitants nomment en diola un païs (une jaunisse ${ }^{5}$ ) ou un ejuusa (un «palu ${ }^{6}$ ), les proches appliquent le plus souvent des remèdes traditionnels.

En outre, le recours à l'automédication, qu'elle soit traditionnelle ou allopathique, présente un intérêt indéniable, celui d'être peu onéreux. En milieu rural africain, le coût des soins représente en effet un obstacle important aux consultations. D'après les déclarations des répondants sur la durée de la maladie, près de la moitié des 134 décès survenus après 60 ans auraient eu lieu suite à une maladie de plus d'un an. Or, les malades constituent une charge pour leur entourage ; les traitements médicaux coûtent chers et deviennent problématiques à payer à moyen ou long terme.

À Mlomp comme ailleurs, les relations entretenues entre le soigné et le soignant sont essentielles, on va consulter l'infirmière du dispensaire parce qu'on la connaît et qu'on lui fait confiance. Il en est de même pour les guérisseurs parmi lesquels le choix est abondant. Enfin, les hôpitaux sont des lieux impersonnels où l'on ne connaît pas le personnel soignant, et qui nécessitent aussi la solidarité

5. Dans le terme français de «maladie », nous devons tenir compte des trois sens bien distingués en anglais : illness (les symptômes ressentis par le malade), disease (l'affection médicalement définie) et sickness (le statut de malade dans la société) (Fassin, 1990). Certains mots ne trouvent pas d'équivalence entre deux langues ou alors une équivalence peu précise, ce qui peut entraîner des confusions et une incompréhension du diagnostic (Jaffré, 1990). païs désigne la coloration jaune du corps donc une jaunisse mais il est parfois traduit par les habitants par fièvre jaune.

6. De la même manière, un ejuusa est un ensemble de symptômes (fièvre, vomissements, diarrhée) que les habitants désignent aussi avec le terme «palu », se référant au paludisme mais cette équivalence est inexacte. 
familiale pour pouvoir héberger les proches, rendre visite au patient, lui apporter son soutien moral mais aussi s'occuper des besoins quotidiens (vêtements, nourriture...) (Kanté et al., 2007). Lorsque la guérison n'est plus envisagée, les malades sont d'ailleurs souvent déshospitalisés et ramenés à leur domicile.

Dans les récits des proches, nous avons identifié les traitements reçus par les personnes durant leur maladie en distinguant synthétiquement quatre types de soins : les soins allopathiques reçus à l'extérieur du domicile (A. ext) comme la consultation dans une case de santé, un dispensaire ${ }^{7}$, un hôpital ou une maternité ; les soins allopathiques reçus à domicile (A. dom) comme la prise de médicaments ou de sirop sans consultation préalable ; les soins traditionnels effectués à l'extérieur du domicile (T. ext) comme la consultation d'un guérisseur, d'un sorcier ou d'un marabout; et enfin les soins traditionnels reçus à domicile (T. dom) telle l'élaboration de tisanes à base de plantes ou de racines sans consultation préalable. Nous avons ensuite regroupé dans une catégorie mixte, les malades qui avaient reçu différents types de soins simultanément ou successivement (allopathique ou traditionnel, externe ou à domicile). Certains malades n'ont reçu aucun soin. Enfin, pour d'autres, l'information n'est pas connue.

Pour les individus décédés entre 60 et 80 ans, les parcours mixtes ont été les plus fréquents $(54 \%)$, mais le recours aux traitements seulement traditionnels, avec ou sans consultation, est aussi important $(20 \%)$ et un malade sur dix n'a reçu aucun soin (Tableau 6). Après 80 ans, plus d'un tiers des malades n'ont reçu aucun soin, ce qui est le plus fréquent avant les parcours de soins mixtes puis traditionnels. Le recours exclusif aux services de santé ne concerne que trois individus sur les 38 pour lesquels on a pu recueillir l'information. Or, ce recours est bien plus fréquent dans les parcours de soins des personnes décédées avant l'âge de 60 ans et en particulier chez les jeunes enfants (Annexe 1). L'automédication par des soins allopathiques à domicile n'a été citée qu'une seule fois, mais ce recours n'a pas non plus été déclaré aux autres âges.

Malgré les faibles effectifs, une différence de soins s'observe entre les hommes et les femmes, en particulier chez les moins de 80 ans (Tableau 6) : les femmes ont consulté les services de santé plus fréquemment que les hommes (respectivement $20 \%$ et $6 \%$ ). Les femmes, même âgées, seraient donc plus proches des services de santé que les hommes. Au village, le dispensaire existe au village depuis 1961 et il se peut que les femmes entretiennent un lien avec les services de santé établi de longue date, lorsqu'elles étaient enceintes et mères de jeunes enfants. Les parcours de soins des hommes sont plus souvent mixtes. Au total, près de 6 hommes sur 10 ont cumulé ou fait succéder plusieurs types de soins. Étant donné les plus fortes proportions de décès de cause mal définie chez les femmes, on aurait pu penser que l'absence de soins concernerait plus ces dernières. Or il n'en est rien.

7. Dans cette catégorie, nous avons aussi considéré les quelques cas où l'infirmière se déplaçait au domicile du malade. 
Tableau 6 - Type de soins reçus par les hommes et les femmes décédés après 60 ans suite à une maladie (Mlomp, 2001-2003)

\begin{tabular}{|c|c|c|c|c|c|c|c|c|c|c|c|c|c|c|c|c|c|c|}
\hline \multirow[b]{3}{*}{$\begin{array}{l}\text { Type } \\
\text { de } \\
\text { soins }\end{array}$} & \multicolumn{6}{|c|}{ Hommes } & \multicolumn{6}{|c|}{ Femmes } & \multicolumn{6}{|c|}{ Ensemble } \\
\hline & \multicolumn{2}{|c|}{$60-79$} & \multicolumn{2}{|c|}{80 et +} & \multicolumn{2}{|c|}{ Total } & \multicolumn{2}{|c|}{$60-79$} & \multicolumn{2}{|c|}{80 et +} & \multicolumn{2}{|c|}{ Total } & \multicolumn{2}{|c|}{$60-79$} & \multicolumn{2}{|c|}{80 et +} & \multicolumn{2}{|c|}{ Total } \\
\hline & $\mathbf{N}$ & $\%$ & $\mathbf{N}$ & $\%$ & $\mathbf{N}$ & $\%$ & $\mathbf{N}$ & $\%$ & $\mathbf{N}$ & $\%$ & $\mathbf{N}$ & $\%$ & $\mathbf{N}$ & $\%$ & $\mathbf{N}$ & $\%$ & $\mathbf{N}$ & $\%$ \\
\hline A. ext & 4 & 7 & 0 & 0 & 4 & 6 & 10 & 24 & 3 & 13 & 13 & 20 & 14 & 15 & 3 & 8 & 17 & 13 \\
\hline $\begin{array}{l}\text { A. } \\
\text { dom }\end{array}$ & 0 & 0 & 0 & 0 & 0 & 0 & 0 & 0 & 1 & 4 & 1 & 2 & 0 & 0 & 1 & 3 & 1 & 1 \\
\hline T. ext & 3 & 6 & 1 & 6 & 4 & 6 & 3 & 7 & 3 & 13 & 6 & 9 & 6 & 6 & 4 & 10 & 10 & 7 \\
\hline T. dom & 7 & 13 & 2 & 13 & 9 & 13 & 5 & 12 & 3 & 13 & 8 & 12 & 12 & 13 & 5 & 13 & 17 & 13 \\
\hline Mixte & 35 & 65 & 6 & 38 & 41 & 59 & 14 & 33 & 5 & 22 & 19 & 30 & 49 & 52 & 11 & 28 & 60 & 45 \\
\hline $\begin{array}{l}\text { Pas de } \\
\text { soins }\end{array}$ & 5 & 9 & 6 & 38 & 11 & 16 & 4 & 10 & 8 & 35 & 12 & 19 & 9 & 9 & 14 & 36 & 23 & 17 \\
\hline Inconnu & 0 & 0 & 1 & 6 & 1 & 1 & 5 & 12 & 0 & 0 & 5 & 8 & 5 & 5 & 1 & 3 & 6 & 4 \\
\hline Total & 54 & 100 & 16 & 100 & 70 & 100 & 42 & 100 & 23 & 100 & 64 & 100 & 95 & 100 & 39 & 100 & 134 & 100 \\
\hline
\end{tabular}

Source : autopsies verbales de Mlomp réalisées de 2002 à 2004.

Il est également possible de détailler les types de soins donnés en premier ou en dernier recours (Tableau 7 et 8). Près de la moitié des malades âgés entre 60 et 80 ans ont reçu un premier soin en consultation dans une infrastructure sanitaire, ce qui est encore le cas pour près d'un malade sur trois au-delà de 80 ans. Mais lorsqu'on observe le dernier soin reçu, la part de personnes exclusivement soignées dans un service de santé au moment du décès diminue au profit d'un arrêt des soins pour les 60-79 ans ou d'un recours mixte pour les plus de 80 ans. Si l'absence de soins est plus fréquente chez les plus âgés, elle ne se cumule donc pas pour autant avec un arrêt des soins pour les individus soignés au départ, comme dans le cas des moins de 80 ans. On peut également voir que l'arrêt des soins allopathiques vers les soins traditionnels ou l'arrêt total des soins est plus fréquent chez les hommes que chez les femmes avant l'âge de 80 ans. Au delà, les effectifs sont trop faibles pour pouvoir interpréter les variations entre le premier et le dernier soin. 
Tableau 7 - Type des premiers soins reçus par les hommes et les femmes décédés après 60 ans suite à une maladie (Mlomp, 2001-2003)

\begin{tabular}{|c|c|c|c|c|c|c|c|c|c|c|c|c|c|c|c|c|c|c|}
\hline \multirow[b]{3}{*}{$\begin{array}{l}\text { Type } \\
\text { de } \\
\text { soins }\end{array}$} & \multicolumn{6}{|c|}{ Hommes } & \multicolumn{6}{|c|}{ Femmes } & \multicolumn{6}{|c|}{ Ensemble } \\
\hline & \multicolumn{2}{|c|}{$60-79$} & \multicolumn{2}{|c|}{80 et +} & \multicolumn{2}{|c|}{ Total } & \multicolumn{2}{|c|}{$60-79$} & \multicolumn{2}{|c|}{80 et +} & \multicolumn{2}{|c|}{ Total } & \multicolumn{2}{|c|}{\begin{tabular}{|l|}
$60-79$ \\
\end{tabular}} & \multicolumn{2}{|c|}{80 et +} & \multicolumn{2}{|c|}{ Total } \\
\hline & $\mathbf{N}$ & $\%$ & $\mathbf{N}$ & $\%$ & $\mathbf{N}$ & $\%$ & $\mathbf{N}$ & $\%$ & $\mathbf{N}$ & $\%$ & $\mathbf{N}$ & $\%$ & $\mathbf{N}$ & $\%$ & $\mathbf{N}$ & $\%$ & $\mathbf{N}$ & $\%$ \\
\hline A. ext & 23 & 43 & 3 & 20 & 26 & 38 & 17 & 47 & 8 & 35 & 25 & 42 & 40 & 44 & 11 & 29 & 51 & 40 \\
\hline $\begin{array}{l}\text { A. } \\
\text { dom }\end{array}$ & 0 & 0 & 0 & 0 & 0 & 0 & 0 & 0 & 1 & 4 & 1 & 2 & 0 & 0 & 1 & 3 & 1 & 1 \\
\hline T. ext & 8 & 15 & 3 & 20 & 11 & 16 & 6 & 17 & 3 & 13 & 9 & 15 & 14 & 16 & 6 & 16 & 20 & 16 \\
\hline T. dom & 12 & 22 & 2 & 13 & 14 & 20 & 6 & 17 & 3 & 13 & 9 & 15 & 18 & 20 & 5 & 13 & 23 & 18 \\
\hline Mixte & 6 & 11 & 1 & 7 & 7 & 10 & 3 & 8 & 0 & 0 & 3 & 5 & 9 & 10 & 1 & 3 & 10 & 8 \\
\hline $\begin{array}{l}\text { Pas de } \\
\text { soins }\end{array}$ & 5 & 9 & 6 & 40 & 11 & 16 & 4 & 11 & 8 & 35 & 12 & 20 & 9 & 10 & 14 & 37 & 23 & 18 \\
\hline Inconnu & 0 & & 1 & & 1 & & 5 & & 0 & & 5 & & 5 & & 1 & & 6 & \\
\hline Total & 54 & 100 & 16 & 100 & 70 & 100 & 41 & 100 & 23 & 100 & 64 & 100 & 95 & 100 & 39 & 100 & 134 & 100 \\
\hline
\end{tabular}

Source : autopsies verbales de Mlomp réalisées de 2002 à 2004.

\section{Le point de vue des proches sur la vieillesse et la mort}

Pour la passation du questionnaire et autopsie verbale, l'enquêteur doit trouver la personne (parente ou proche) la mieux renseignée. Le répondant a donc de grande chance d'être la personne qui a soigné le malade avant sa mort. En étudiant le lien de parenté entre le répondant et le défunt, on s'aperçoit d'une distinction importante entre les hommes et les femmes (Tableau 9). Sans tenir compte des époux qui répondent pour leur conjoint, ce sont majoritairement des hommes qui répondent pour les hommes, le sexe du répondant étant plus mixte pour les femmes. Parmi les enfants, ce sont beaucoup plus fréquemment les fils qui répondent pour leur père et les filles pour leur mère. Dans la mesure où l'on sait que les femmes, lorsqu'elles se marient, rejoignent la concession de leur époux, on peut supposer que, tandis que les fils vivent avec leurs parents, ce n'est pas le cas des filles. Nous avons relevé par ailleurs que le fait d'avoir des garçons pour les femmes avait un effet protecteur sur leur mortalité, tandis que pour les hommes, leur mortalité n'était pas affectée par leur descendance (Duthé et al., 2008). Ces éléments suggèrent que des différences de genre peuvent exister en matière de prise en charge, au détriment des femmes âgées. Pour autant, il est difficile de conclure parce que l'enquêteur peut introduire un bais par le choix présupposé d'aller 
questionner la fille d'une mère et le fils d'un père sur les histoires des maladies et des symptômes.

Tableau 8 - Type des derniers soins reçus par les hommes et les femmes décédés après 60 ans suite à une maladie (Mlomp, 2001-2003)

\begin{tabular}{|c|c|c|c|c|c|c|c|c|c|c|c|c|c|c|c|c|c|c|}
\hline & \multicolumn{6}{|c|}{ Hommes } & \multicolumn{6}{|c|}{ Femmes } & \multicolumn{6}{|c|}{ Ensemble } \\
\hline & \multicolumn{2}{|c|}{$60-79$} & \multicolumn{2}{|c|}{80 et +} & \multicolumn{2}{|c|}{ Total } & \multicolumn{2}{|c|}{$60-79$} & \multicolumn{2}{|c|}{80 et +} & \multicolumn{2}{|c|}{ Total } & \multicolumn{2}{|c|}{$60-79$} & \multicolumn{2}{|c|}{80 et +} & \multicolumn{2}{|c|}{ Total } \\
\hline $\begin{array}{l}\text { Type } \\
\text { de } \\
\text { soins }\end{array}$ & $\mathbf{N}$ & $\%$ & $\mathbf{N}$ & $\%$ & $\mathbf{N}$ & $\%$ & $\mathbf{N}$ & $\%$ & $\mathbf{N}$ & $\%$ & $\mathbf{N}$ & $\%$ & $\mathbf{N}$ & $\%$ & $\mathbf{N}$ & $\%$ & $\mathbf{N}$ & $\%$ \\
\hline A. ext & 14 & 26 & 0 & 0 & 14 & 20 & 14 & 39 & 2 & 9 & 16 & 27 & 28 & 31 & 2 & 5 & 30 & 23 \\
\hline $\begin{array}{l}\text { A. } \\
\text { dom }\end{array}$ & 0 & 0 & 0 & 0 & 0 & 0 & 0 & 0 & 1 & 4 & 1 & 2 & 0 & 0 & 1 & 3 & 1 & 1 \\
\hline T. ext & 10 & 19 & 3 & 20 & 13 & 19 & 4 & 11 & 3 & 13 & 7 & 12 & 14 & 16 & 6 & 16 & 20 & 16 \\
\hline T. dom & 10 & 19 & 2 & 13 & 12 & 17 & 6 & 17 & 3 & 13 & 9 & 15 & 16 & 18 & 5 & 13 & 21 & 16 \\
\hline Mixte & 6 & 11 & 3 & 20 & 9 & 13 & 5 & 14 & 4 & 17 & 9 & 15 & 11 & 12 & 7 & 18 & 18 & 14 \\
\hline $\begin{array}{l}\text { Pas de } \\
\text { soins }\end{array}$ & 14 & 26 & 7 & 47 & 21 & 30 & 7 & 19 & 10 & 43 & 17 & 29 & 21 & 23 & 17 & 45 & 38 & 30 \\
\hline Inconny & 0 & & 1 & & 1 & & 5 & & 0 & & 5 & & 5 & & 1 & & 6 & \\
\hline Total & 54 & 100 & 16 & 100 & 70 & 100 & 41 & 100 & 23 & 100 & 64 & 100 & 95 & 100 & 39 & 100 & 134 & 100 \\
\hline
\end{tabular}

Source : autopsies verbales de Mlomp réalisées de 2002 à 2004.

Ces résultats doivent être interprétés avec précaution, parce que cette source est baisée au sens où elle révèle des comportements de personnes qui sont par la suite décédées et non de celles qui ont survécu à une maladie, des informations qui sont en plus recueillies auprès d'une tierce personne. Cependant, ils confirment au niveau local ce qui a déjà été relevé par ailleurs : plus l'âge des malades est élevé, moins les soins de type allopathique sont courants et moins le recours aux soins de manière générale est fréquent. Les personnes âgées n'ont pas la même éducation sanitaire que les plus jeunes adultes qui ont pu aller à l'école ou qui se rendent régulièrement au dispensaire comme parents de jeunes enfants. Les seniors sont plus éloignés du corps médical et la médecine allopathique ne fait pas forcément sens. Enfin, l'absence de soins révèle également un rapport à la maladie et à la mort particulier pour les plus âgés. L'étude des discours tenus par les proches pour raconter l'histoire de la maladie ayant conduit au décès permet de mieux comprendre les absences ou les arrêts de soins observés. En premier lieu, la vieillesse est invoquée de manière récurrente dans les récits, surtout après 80 ans 
Tableau 9-Lien de parenté du répondant à l'autopsie verbale pour les hommes et les femmes décédés après 60 ans suite à une maladie (Mlomp, 2001-2003)

\begin{tabular}{|c|c|c|c|c|c|c|c|c|c|c|c|c|c|c|c|c|c|c|}
\hline \multirow[b]{3}{*}{$\begin{array}{l}\text { Lien } \\
\text { de } \\
\text { parenté }\end{array}$} & \multicolumn{6}{|c|}{ Hommes } & \multicolumn{6}{|c|}{ Femmes } & \multicolumn{6}{|c|}{ Ensemble } \\
\hline & \multicolumn{2}{|c|}{$60-79$} & \multicolumn{2}{|c|}{80 et +} & \multicolumn{2}{|c|}{ Total } & \multicolumn{2}{|c|}{$60-79$} & \multicolumn{2}{|c|}{80 et +} & \multicolumn{2}{|c|}{ Total } & \multicolumn{2}{|c|}{$60-79$} & \multicolumn{2}{|c|}{80 et +} & \multicolumn{2}{|c|}{ Total } \\
\hline & $\mathbf{N}$ & $\%$ & $\mathbf{N}$ & $\%$ & $\mathbf{N}$ & $\%$ & $\mathbf{N}$ & $\%$ & $\mathbf{N}$ & $\%$ & $\mathbf{N}$ & $\%$ & $\mathbf{N}$ & $\%$ & $\mathbf{N}$ & $\%$ & $\mathbf{N}$ & $\%$ \\
\hline $\begin{array}{l}\text { Époux, } \\
\text { épouse }\end{array}$ & 11 & 20 & 2 & 13 & 13 & 19 & 9 & 22 & 1 & 4 & 10 & 16 & 20 & 21 & 3 & 8 & 23 & 17 \\
\hline $\begin{array}{l}\text { Sœur, } \\
\text { belle- } \\
\text { sœur, } \\
\text { cousine }\end{array}$ & 1 & 2 & 0 & 0 & 1 & 1 & 1 & 2 & 0 & 0 & 1 & 2 & 2 & 2 & 0 & 0 & 2 & 1 \\
\hline $\begin{array}{l}\text { Frère, } \\
\text { beau- } \\
\text { frère, }\end{array}$ & 6 & 11 & 1 & 6 & 7 & 10 & 4 & 10 & 1 & 4 & 5 & 8 & 10 & 11 & 2 & 5 & 12 & 9 \\
\hline Fille & 6 & 11 & 0 & 0 & 6 & 9 & 10 & 24 & 8 & 35 & 18 & 28 & 16 & 17 & 8 & 21 & 24 & 18 \\
\hline Fils & 16 & 30 & 5 & 31 & 21 & 30 & 8 & 20 & 8 & 35 & 16 & 25 & 24 & 25 & 13 & 33 & 37 & 28 \\
\hline $\begin{array}{l}\text { Belle- } \\
\text { fille, } \\
\text { nièce... }\end{array}$ & 3 & 6 & 1 & 6 & 4 & 6 & 5 & 12 & 2 & 9 & 7 & 11 & 8 & 8 & 3 & 8 & 11 & 8 \\
\hline $\begin{array}{l}\text { Beau- } \\
\text { fils, } \\
\text { neveu... }\end{array}$ & 10 & 19 & 7 & 44 & 17 & 24 & 4 & 10 & 3 & 13 & 7 & 11 & 14 & 15 & 10 & 26 & 24 & 18 \\
\hline Autre & 1 & 2 & 0 & 0 & 1 & 1 & 0 & 0 & 0 & 0 & 0 & 0 & 1 & 1 & 0 & 0 & 1 & 1 \\
\hline Total & 54 & 100 & 16 & 100 & 70 & 100 & 41 & 100 & 23 & 100 & 64 & 100 & 95 & 100 & 39 & 100 & 134 & 100 \\
\hline
\end{tabular}

Source : autopsies verbales de Mlomp réalisées de 2002 à 2004.

(4 fois sur 10) en cause déclarée du décès ou en cause associée. Se dégage également de ces récits une certaine acceptation de la mort comme étant naturelle et irrémédiable, voire un souhait de mourir qui aurait été exprimé par le malade lui-même que l'on retrouve dans cinq récits de personnes décédées après 80 ans, soit plus d'un sur dix ${ }^{8}$. Ainsi, cet homme explique que sa mère est morte de vieillesse (elle avait 89 ans), alors qu'elle avait une plaie infectée :

8. Avant 80 ans, cette volonté de mourir se retrouve dans cinq récits, soit $5 \%$ des décès de ce groupe d'âges. 
« Ma mère est morte de vieillesse. Elle devait partir, d'ailleurs c'est ce qu'elle souhaitait, lors de son palu pour ne pas nous fatiguer. En effet, elle a eu une semaine de fièvre et de maux de tête. Je peux dire que c'est cela la cause du décès, à part la plaie qui se trouvait au niveau de sa cheville qui était très infectée » (autopsie verbale).

Autre exemple, le père de cet homme est décédé à l'âge de 95 ans, il explique que son père était tellement vieux que la mort l'avait «oublié » :

«Mon père était vieux. Lui-même disait que la mort l'a oublié. Du reste, il était malade car il souffrait des articulations. Il se déplaçait difficilement. Mais sa mort ne m'a pas surpris, ma mère m'avait dit de faire attention parce que le vieux était au bout de ses forces. Je suis allé à son chevet et l'ai observé un bon moment et j'ai compris. Le soir, vers $18 \mathrm{~h}$, il rendit l'âme tout tranquillement» (autopsie verbale).

Avec la vieillesse, la mort des personnes âgées s'inscrit dans l'ordre naturel des choses, y compris pour elles, quand elles ne se sentent plus utiles au groupe familial ou social, lorsqu'elles se perçoivent et/ou sont perçues comme une charge de travail et de fatigue pour les proches. Bien sûr, cette conclusion doit être nuancée : dans leurs discours, les proches peuvent aussi justifier le faible investissement dans les soins de leur parent âgé. Ce plus faible recours aux soins, on l'a déjà mentionné, peut aussi être lié à une hiérarchisation contrainte des dépenses du ménage, les soins des aînés n'étant pas prioritaires. Malgré tout, le souhait de mourir chez soi et au sein de sa famille est très prégnant. Sur les 134 individus décédés après 60 ans, $80 \%$ des décès ont d'ailleurs eu lieu au domicile.

\section{Conclusion}

En dehors de la période d'activité agricole, la population de Mlomp comprend une part relativement importante de seniors $(15 \%)$. La structure des ménages ne met pas en évidence de distinction entre les hommes et les femmes. Ces dernières font cependant bien plus fréquemment l'expérience du veuvage que les hommes. Nous avons aussi relevé que les causes de décès des femmes étaient moins souvent déterminées. Dans un système patrilinéaire comme à Mlomp, les femmes âgées pourraient être moins bien prises en charge que leurs pairs masculins. Cette hypothèse doit toutefois être testée par des études complémentaires.

L'analyse des histoires des maladies ayant conduit au décès révèle une diversité des types de soins aux âges élevés, constatée par ailleurs et notamment au Cameroun (Mveing et Fomekong, 2008). Pour les femmes en particulier, le recours exclusif aux services de santé reste encore assez important après 60 ans. L'activité du dispensaire de Mlomp a joué un rôle considérable sur les conditions sanitaires locales, ce qui doit nécessairement avoir des effets positifs sur la santé des adultes, y compris pour les plus âgés. Ouvert en 1961, les villageois qui ont aujourd'hui la cinquantaine connaissent le dispensaire depuis leur enfance. Les anciens de demain se tourneront certainement plus vers le dispensaire que ne le font les aînés d'aujourd'hui. Toutefois, une part importante des personnes âgées décédées à la 
suite d'une maladie n'était pas soignée au moment de leur décès. Les proches peuvent considérer que la mort est naturelle et il n'y a pas lieu de chercher plus loin les raisons de ce décès dû à la vieillesse et donc de tenter une voie de guérison. En outre, dans des situations financières difficiles, on suppose que les soins des personnes âgées ne sont pas prioritaires, surtout s'ils sont malades dans la durée. Mais aussi parce qu'elles le souhaitent, les personnes âgées ne sont pas toujours soignées, préférant mourir chez elles dans leur foyer. Cette étude ne permet pas d'identifier l'ensemble des comportements de soins en population générale : elle a été réalisée sur un petit échantillon de maladies ayant conduit au décès. Les résultats doivent donc être interprétés avec précaution puisque l'analyse ne porte pas sur les épisodes morbides ayant abouti à une guérison, que les informations sont déclaratives et recueillies auprès d'un proche qui a sa propre interprétation des événements passés (omission, justification, etc.). Enfin, nous n'avons pas traité de la mobilité, qui joue un rôle important dans la situation sanitaire des personnes âgées : d'une part du point de vue de l'absence en saison sèche de nombreux jeunes adultes et d'autre part des mobilités pour se soigner en dehors du village (consultation auprès d'un guérisseur, hospitalisation, installation chez un enfant domicilié ailleurs). Une enquête sur la santé et les parcours de soins est donc nécessaire pour conforter ces premiers résultats et distinguer notamment ce qui relève du choix des malades explicité par leurs proches des contraintes potentielles et sous-jacentes aux décisions de soins. Sur ce point, une enquête anthropologique sur la prise en charge des personnes âgées et des malades, de leur statut, des prises de décisions en situation de contrainte économique permettrait également de mieux comprendre les comportements observés quantitativement.

Dans les pays caractérisés par une espérance de vie élevée, les limites de la vie sont sans cesse repoussées, on parle désormais de décès «prématurés » lorsqu'ils surviennent avant l'âge de 65 ans (Jougla, 2003) et on entreprend de combattre la sénescence (Horiuchi, 1999). Le contraste est énorme avec le milieu rural africain où la situation sanitaire des personnes âgées est méconnue. Même dans le cadre d'un suivi de population comme à Mlomp, l'espérance de vie à 60 ans relève d'une estimation et la part des décès de cause mal définie est tellement importante après cet âge qu'il est difficile de dégager des conclusions sur la structure de la mortalité par causes. Face au manque de données, la santé perçue apparaît comme un simple et bon indicateur de la situation sanitaire globale (Kuate Defo, 2005 ; Kuhn et al., 2006). Les sites de suivi démographique font de plus en plus référence dans le domaine de la santé et de la mortalité dans les pays du Sud, ils doivent offrir l'opportunité de travailler sur cette population grandissante. La prise en charge des soins des personnes âgées préoccupe actuellement les états africains, d'autant plus que les seniors sont rarement pourvus d'une pension de retraite. Ainsi, le Sénégal a mis en place le $1^{\text {er }}$ septembre 2006 le plan SESAME pour la gratuité des soins des plus de 60 ans. Il est probable que ce nouveau dispositif améliorera considérablement les conditions de prise en charge des seniors. 
Annexe 1. Type de soins reçus par les individus décédés à la suite d'une maladie par grand groupe d'âge au décès (Mlomp, 2001-2003)

\begin{tabular}{l|c|c|c|c|c|c|c|c|c|c}
\hline \multirow{2}{*}{$\begin{array}{c}\text { Type } \\
\text { de soins }\end{array}$} & \multicolumn{2}{|c|}{ Avant 5 ans } & \multicolumn{3}{|c|}{$5-59$ ans } & \multicolumn{2}{c|}{$60-79$ ans } & \multicolumn{2}{c|}{80 ans et + } & \multicolumn{2}{c}{ Total } \\
\cline { 2 - 12 } & N & $\%$ & N & $\%$ & N & $\%$ & N & $\%$ & N & $\%$ \\
\hline A. ext & 25 & 64 & 18 & 30 & 14 & 16 & 3 & 8 & 54 & 27 \\
\hline A. dom & 0 & 0 & 0 & 0 & 0 & 0 & 1 & 3 & 1 & 0 \\
\hline T. ext & 0 & 0 & 3 & 5 & 6 & 7 & 4 & 10 & 13 & 6 \\
\hline T. dom & 1 & 3 & 3 & 5 & 12 & 13 & 5 & 13 & 21 & 9 \\
\hline Mixte & 11 & 28 & 34 & 58 & 49 & 54 & 11 & 29 & 105 & 46 \\
\hline $\begin{array}{l}\text { Pas de } \\
\text { soins }\end{array}$ & 2 & 5 & 1 & 2 & 9 & 10 & 14 & 37 & 26 & 12 \\
\hline Inconnu & 3 & - & 5 & - & 5 & - & 1 & - & 14 & - \\
\hline Total & 42 & 100 & 64 & 100 & 95 & 100 & 39 & 100 & 240 & 100 \\
\hline
\end{tabular}

Source : autopsies verbales de Mlomp réalisées de 2002 à 2004. 


\section{Bibliographie}

Adjuik M., Smith T., Clarck S. et al. [2006], « Cause specific mortality rates in sub-Saharan Africa and Bangladesh », Bulletin of the WHO, vol. 84, $\mathrm{n}^{\circ}$ 3, p. 181-188.

Antolne P. (éd) [2007, Les relations intergénérationnelles en Afrique. Approche plurielle, Paris, Ceped, $255 \mathrm{p}$.

Attias-Donfut C., Rosenmayr L. [1994], « Avant-propos », in Attias-Donfut C., RosenMAYR L. (dir), Vieillir en Afrique, Paris, Puf, p. 13-15.

CENTRE INTERNATIONAL DE GÉRONTOLOGIE SOCIALE. [1985], « Recommandations adoptées par la Conférence africaine de la gérontologie, Dakar, 10-14 décembre », Paris, Centre international de gérontologie sociale, $77 \mathrm{p}$.

Chandramohan D., Soleman N., Shibuya K., Porter J., [2005], «Ethical issues in the application of verbal autopsies in mortality surveillance systems », Tropical Medicine and International Health, vol. 10, no 11, p. 1087-1089.

DutHÉ G. [2006], «La transition sanitaire en milieu rural sénégalais. Évolution de la mortalité à Mlomp depuis 1985 et influence du paludisme chimiorésistant », Thèse de doctorat de démographie, Paris, Muséum national d'histoire naturelle, $350 \mathrm{p}$.

DutHÉ G. [2008], «Recrudescence du paludisme au Sénégal : la mesure de la mortalité palustre à Mlomp », Population, vol. 63, nº 3, p. 505-530.

Duthé G., Pison G., LAuREnT R. [2008], « Long term effects of reproductive history on mortality among women and men in a rural area of Senegal », Congrès américain de population, PAA, 17-19 avril, Nouvelle Orléans (poster).

Duthé G., LAURent R., Pison G. [2009], «Vivre et mourir après 60 ans en milieu rural africain », Paris, Ined (Document de travail $\mathrm{n}^{\circ} 160$ ), $25 \mathrm{p}$.

ENel C., Pison G. [2007], «Veuvage et lévirat: une étude de cas à Mlomp (Sénégal) » in Locoh T. (dir), Genre et sociétés en Afrique, Paris, Ined (Les Cahiers de l'Ined, nº 160), p. 327-341.

FASSIN D. [1990], «Maladie et médecines », in FASSIN D., JAFFre Y. (éd), Sociétés, développement et santé, Paris, Ellipses, p. 38-49.

FAUVEAU V. [2006], « Assessing probable causes of death without death registration or certificates : a new science ?», Bulletin of the WHO, vol. 84, $\mathrm{n}^{\circ} 3$, p. 246-247.

Gajalakshmi V., Peto R. [2006], « Commentary : Verbal autopsy procedure for adult deaths », International Journal of Epidemiology, vol. 35, $\mathrm{n}^{\circ} 3$, p. 758-759.

Garenne M., Fauveau V. [2006], «Potential and limits of verbal autopsies », Bulletin of the $W H O$, vol. 84, n 3, p. 164-165.

HiLL K.H. [1999], « The measurement of adult mortality : an assessment of data availability, data quality and estimation methods », in Chamie J., Cliquet R. (éd), Health and Mortality Issues of Global Concern, Bruxelles/New York, Flemish Scientific Institute/United Nations, p. $72-83$.

Horiuchi S. [1999], «Epidemiological transitions in human history », in Chamie J., CliqueT R. (éd) Health and Mortality Issues of Global Concern, B Bruxelles/New York, Flemish Scientific Institute/United Nations, p. 54-71.

JAFFRE Y. [1990], « Comprendre. Les mots du malades », in FASSIN D., JAFFRE Y. (éd.) Sociétés, développement et santé, Paris, Ellipses, p. 126-133.

Jougla E. [2003], «La mortalité prématurée en France : Editorial », BEH, n 30-31, p. 133. 
KA-Cisse M., Sow A. M. [1995], « La prise en charge sanitaire des personnes âgées. Des besoins encore mal compris », PopSahel, n ${ }^{\circ} 22$, p. 20-22.

Kahn K., Tollman S., Thorogood M. et al. [2006], «Older adults and the health transition in Agincourt, rural south Africa: New understanding, growing complexity », National Research Council, "Aging in Sub-Saharan Africa: Recommendations for Furthering Research », Washington, DC : The National Academies Press, p. 314-341.

Kaufman J., Asuzu M., Rotimi C. et al. [1997], «The absence of adult mortality data for sub-Saharan Africa : a practical solution », Bulletin of the WHO, n ${ }^{\circ}$ 5, p. 389-395.

Kante M., Guyavarch E., Pison G. [2007], «L'offre sanitaire en Afrique correspond-t-elle aux besoins des populations? Le cas de la construction d'un nouvel hôpital dans le milieu rural au Sénégal », Conférence de la population africaine, UEPA, 10-14 décembre, Arusha (communication).

Kouame A. [1990], Le vieillissement de la population en Afrique, Ottawa, Centre de Recherches pour le Développement International, $46 \mathrm{p}$.

Kowal P., Dowd J., Wolfson L. [2000], «Creating a minimum data set on ageing in subSaharan Africa », Southern African Journal of Gerontology, $\mathrm{n}^{\circ}$ 9, p. 18-23.

Kowal p. et al. [2002], « Data for older adult populations in sub-Saharan Africa » (correspondence), The Lancet, n ${ }^{\circ} 360$, p. 91.

KuAte-Defo B. [2005], «Facteurs associés à la santé perçue et à la capacité fonctionnelle des personnes âgées dans la préfecture de Bandjoun au Cameroun », Cahiers québécois de démographie, vol. $34, \mathrm{n}^{\circ} 1$, p. 1-46.

Kuhn R., Rahman O., MenKen J. [2006], « Survey measures of health : how well do selfreported and observed indicators measure health and predict mortality? », National Research Council, Aging in Sub-Saharan Africa: Recommendations for Furthering Research, Washington, DC, The National Academies Press, p. 314-341.

LocoH T., MAKDESSI Y. [2002], «Transition démographique et statut des personnes âgées en Afrique, quelles perspectives? », Vivre plus longtemps, avoir moins d'enfants, quelles implications?, Actes du $10^{\mathrm{e}}$ colloque international de l'AIDELF, 10-13 octobre 2002, ByblosJbeil, Liban. Paris, Puf, p. 615-626.

MAssengo S. [2002], «Poverty and health in old age in Africa: issues and programmatic response », Population Ageing and Development. Social, Health and Gender Issues. Population and Development Strategies, n ${ }^{\circ} 3$, p. 41-42.

Mveing S., FomeKong F. [2008], « Conditions de vie des ménages et recours aux soins parmi les personnes âgées au Cameroun», Étude de la Population Africaine, vol. 23, p. 1, p. 85-102.

National Research Council [2006], Aging in Sub-Saharan Africa: Recommendations for Furthering Research, Washington, DC, The National Academies Press, 356 p.

Nations Unies [2009], World Population Prospects. The 2008 revision, New York, Division de la Population, Département des affaires économiques et sociales des Nations Unies.

Ndiaye S., Ayad M. [2006], Enquête Démographique et de Santé au Sénégal 2005, Calverton, Maryland, USA : Centre de Recherche pour le Développement Humain [Sénégal] et ORC Macro.

OlIVIER DE SARDAN J.-P. [1999], « Les entités nosologiques populaires internes : quelques logiques représentationnelles », in JAFFRE Y., OLIVIER DE SARDAN J.-P. (dir), La construction sociale des maladies, Paris, Puf, p. 71-87.

OMS [1977], Classification internationale des maladies, $9^{e}$ révision (1975), Genève, OMS. 
ONUSIDA [2008], Rapport sur l'épidémie mondiale de sida, Genève : ONUSIDA.

PISON G. [2005], «Population observatories as sources of information on mortality in developing countries », Demographic Research, n ${ }^{\circ} 13$, p. 301-334.

Pison G., Trape J.-F., Lefebvre M., Enel C. [1993], « Rapid decline in child mortality in a rural area of Senegal », International Journal of Epidemiology, vol. 22, $\mathrm{n}^{\circ}$ 1, p. 72-80.

Pison G., Kodio B., Guyavarch E., Etard J.-F. [2000], « La mortalité maternelle en milieu rural au Sénégal », Population, nº 6, p. 1003-1018.

Pison G., Wade A., Gabadinho A., Enel C. [2002], «Mlomp DSS, Senegal », in Indepth network. Population and Health in Developing Countries (Volume 1), Ottawa, International development research centre, p. 271-278.

Pison G., Duthe G., Guyavarch E., Marra A., Chippaux J.-P. [2005], « La mortalité violente au Sénégal : niveaux et causes dans trois zones rurales », Congrès international de la population, UIESP, 18-23 juillet, Tours, France (communication).

Prost A. [2000], «L'Afrique n'est pas un cas particulier. Retour des grands fléaux, émergence de maladies nouvelles », in Gruenais M.-E., Pourtier R. (dir), «La santé en Afrique. Anciens et nouveaux défis ", Afrique contemporaine, $n^{\circ}$ 195, p. 15-23.

Renaut C. [1994], "L'Afrique au sud du Sahara : quelques éléments de démographie », in Attias-Donfut C., Rosenmayr L. (dir) Vieillir en Afrique, Paris, Puf.

Sankoh O., Kynast-Wolf G., Kouyate B., Becher H. [2003], « Patterns of adult and old-age mortality in rural Burkina Faso », Journal of Public Health Medicine, vol. 25, $\mathrm{n}^{\circ} 4$, p. 372-376.

Schieber G., Maeda A. [1999], «Health Care Financing And Delivery In Developing Countries », Health Affairs, vol. 18, nº 3, p. 193-205.

SCHOUMAKeR B. [2000], «Le vieillissement en Afrique subsaharienne », Espace Populations Sociétés, $\mathrm{n}^{\circ} 3$, p. 379-390.

SHRESTHA L. [2000], « Population aging in developing countries », Health Affairs, vol. 19, $\mathrm{n}^{\circ} 3$, p. 204-212.

TABUTIN D. [1987], «Quelques perspectives des populations âgées dans les pays jeunes », Espace Populations Sociétés, $\mathrm{n}^{\mathrm{o}} 2$ 2, p. 365-374.

Thomas L.V. [1994], «Vieillesse et mort en Afrique », in Attias-Donfut C., Rosenmayr L. (dir), Vieillir en Afrique, Paris, Puf, p. 149-167.

Timeus I., JASSEH M. [2004], «Adult mortality in sub-Saharan Africa : evidence from Demographic and health surveys », Demography, vol. 41, n 4, p. 757-772.

Velkoff V., Kowal p. [2007], Population aging in sub-saharan Africa : demographic dimensions, International Population Reports, P95/07-1, Washington DC, US Census bureau.

ZimMER Z., DAYTON J. [2005], « Older adults in sub-Saharan Africa living with children and grandchildren », Population Studies, vol. 59, n 3, p. 295-312. 